\title{
A NOTE ON AN INEQUALITY OF E. SCHMIDT
}

\author{
RICHARD BELLMAN
}

In a note inserted in the Sitzungsberichte der Preussicher Akademie, 1932, E. Schmidt stated without proof a relation

$$
\left[\int_{-1}^{1} f^{\prime}(x)^{2} d x\right]^{1 / 2} \leqq k_{N} N^{2}\left[\int_{-1}^{1} f(x)^{2} d x\right]^{1 / 2}
$$

where $f(x)$ is a polynomial of degree $N$, and it was subsequently shown by Hille, Szego and Tamarkin that $k_{N}$ is a bounded function of $N$ such that $\lim _{N \rightarrow \infty} k_{N}=\pi^{-1}$.

In a joint paper, generalizing the classical Markoff theorem

$$
\max _{-1 \leqq x \leqq 1}\left|f^{\prime}(x)\right| \leqq N^{2} \max _{-1 \leqq x \leqq 1}|f(x)|
$$

to general mean values of the form $\int_{-1}^{1}|f(x)|^{p} d x, p \geqq 1$, Hille, Szego and Tamarkin [1] ${ }^{1}$ gave three proofs of the inequality (1), two products of the general case of exponent $p$, and another for the case $p=2$, stated by the authors to be similar to the original unpublished proof of Schmidt.

It seems worthwhile to sketch a short elementary proof of the important case $p=2$, depending only upon an elementary inequality : ${ }^{2}$

$$
n \sum_{1}^{n} a_{k}^{2}-\left(\sum_{1}^{n} a_{k}\right)^{2} \geqq 0
$$

and a simple property of the Legendre polynomials.

The required result for Legendre polynomials is [2] $P_{n+1}^{\prime}(z)$ $-P_{n-1}(z)=(2 n+1) P_{n}(z)$, where $P_{n}(z)$ is the $n$th Legendre polynomial. From this we obtain

$$
\begin{aligned}
P_{2 n}^{\prime} & =\sum_{0}^{n-1}(4 k+3) P_{2 k+1}, \\
P_{2 n+1}^{\prime} & =\sum_{0}^{n}(4 k+1) P_{2 k} .
\end{aligned}
$$

Let the polynomial of the $N$ th degree be expressed in Legendre polynomials

Received by the editors January 31,1944 .

${ }^{1}$ Numbers in brackets refer to the references cited at the end of the paper.

2 The use of this inequality, instead of a more complicated one due to Hardy, was suggested by Professor Szász. It simultaneously simplifies the proof and yields a better constant than that originally obtained by the author. 


$$
f_{N}(x)=\sum_{k=0}^{N} a_{k} P_{k}(x) .
$$

Then, using the well known orthogonality relations of the $P_{n}$,

$$
\int_{-1}^{1} f_{N}(x)^{2} d x=2 \sum_{0}^{N} \frac{a_{k}^{2}}{2 k+1}
$$

Using (3),

Thus

$$
\begin{aligned}
f_{N}^{\prime}(x)= & \sum_{2 k \leqq N-1}(4 k+1) P_{2 k}(x)\left[\sum_{n \geqq k+1} a_{2 n+1}\right] \\
& +\sum_{2 k \leqq N-1}(4 k+3) P_{2 k+1}(x)\left[\sum_{n \geqq k+1} a_{2 n}\right] .
\end{aligned}
$$

$$
\begin{aligned}
\int_{-1}^{1} f_{N}^{\prime}(x)^{2} d x=2 \sum_{2 k \leqq N-1}(4 k+1)\left[\sum_{r \geqq k} a_{2 r+1}\right]^{2} \\
+2 \sum_{2 k \leqq N-1}(4 k+3)\left[\sum_{r \geqq k} a_{2 n}\right]^{2} .
\end{aligned}
$$

Let $a_{r}=((2 r+1) / 2)^{1 / 2} b_{r}$. Then the first sum is

$$
\begin{aligned}
2 \sum_{2 k \leqq N-1}(4 k+1) & {\left[\sum_{r \geqq k} a_{2 r+1}\right]^{2} } \\
& \leqq 2\left[\sum_{2 k \leqq N-1}(4 k+1)\right]\left[\sum\left(\frac{4 k+1}{2}\right)^{1 / 2} b_{2 k+1}\right]^{2} \\
& \leqq \frac{N(N+1)(2 N+1)}{4}\left[\sum b_{2 k+1}\right]^{2} \\
& \leqq(N+1)^{4} / 2\left[\sum b_{2 k+1}^{2}\right]
\end{aligned}
$$

upon using the inequality (2) upon $\left[\sum b_{2 k+1}\right]^{2}$.

The same process yields the same estimate for the second sum. Thus

$$
\begin{aligned}
\int_{-1}^{1} f_{N}^{\prime}(x)^{2} d x \leqq \frac{(N+1)^{4}}{2} \sum_{0}^{N} b_{k}^{2} & =\frac{(N+1)^{4}}{2} \sum_{0}^{N} \frac{2 a_{k}^{2}}{2 k+1} \\
& =\frac{(N+1)^{4}}{2} \int_{-1}^{1} f_{N}(x)^{2} d x
\end{aligned}
$$

This finishes the proof of (1) and yields lim sup $k_{N} \leqq 1 / 2^{1 / 2}$. 
It is clear from the proof that by using Laguerre and Hermite polynomials with the corresponding weight functions, one can obtain similar results for the intervals $(0, \infty)$ and $(-\infty, \infty)$.

\section{REFERENCES}

1. E. Hille, G. Szego, A. Tamarkin, On some generalizations of a theorem of A. Markoff, Duke Math. J. vol. 3 (1937) p. 729.

2. Whittaker-Watson, Modern analysis, Cambridge, 1935, p. 307 (IV).

Princeton UnIVERSITY

\section{TRANSCENDENCE OF CERTAIN CONTINUED FRACTIONS}

\section{G. CUTHBERT WEBBER}

Even today very little is known about which regular continued fraction expansions represent transcendental numbers. In 1851 Liouville $^{1}$ proved the transcendence of the numbers named after him, in the expansions of which the partial quotients increase very rapidly; in 1906 Maillet $^{2}$ proved the transcendence of certain expansions in which the partial quotients may be bounded. C. L. Siegel ${ }^{3}$ proved that if the partial quotients of the expansion form an arithmetic progression of order one, the number represented is transcendental. In this paper well known transformations of continued fractions are applied, in conjunction with the Siegel theorem, to prove the transcendence of certain classes of continued fraction expansions.

We shall use the notation

$$
b_{0}+\frac{a_{1}}{b_{1}}+\frac{a_{2}}{b_{2}}+\cdots
$$

to represent a continued fraction expansion, with the partial numerators and denominators $a_{j}$ and $b_{j}$ respectively. The $j$ th convergent is denoted by $A_{j} / B_{j}$. When the expansion is regular the following notations are also used:

Received by the editors April 6, 1944.

${ }^{1} \mathrm{~J}$. Liouville, Sur des classes très-étendues de quantités dont la valeur n'est ni algêbrique, $n$ même reductible d̀ des irrationnelles algébrique, J. Math. Pures Appl. vol. 16 (1851).

2 E. Maillet, Introduction a la théorie des nombres transcendants, Paris, 1906.

3 C. L. Siegel, Über einige Anwendungen diophantischer Approximationen, Abhandlung der Preussischen Akademie der Wissenschaften, Physikalische-Mathematische Klasse, no. 1 (1929) p. 29. 